Andréia Ramos ${ }^{1}$

João Ferreira da Silva Filho

Silvia Rodrigues Jardim ${ }^{3}$

\section{Dados sociodemográficos e condições de trabalho de pintores expostos a solventes em uma universidade pública da cidade do Rio de Janeiro*}

\author{
Sociodemographic data and working conditions of painters \\ exposed to solvents at a public university in Rio de Janeiro
}

\begin{abstract}
${ }^{1}$ Psiquiatra do CEREST/Juiz de Fora. Colaboradora do Programa Organização do Trabalho e Saúde Mental (OTSAM)/IPUB/UFRJ. Professora do Centro de Ensino Superior de Juiz de Fora e da Sociedade Universitária Redentor.

2 Professor Titular da Universidade Federal do Rio de Janeiro. Médico Psiquiatra do IPUB da Universidade Federal do Rio de Janeiro, Programa Organização do Trabalho e Saúde Mental (OTSAM)

${ }^{3}$ Coordenadora do Centro de Referência em Saúde Mental do Trabalhador (CRESAMT) da Universidade Federal do Rio de Janeiro. Médica Psiquiatra do IPUB

\section{Resumo}

O artigo apresenta os dados sociodemográficos e as condições de trabalho de pintores de uma universidade pública da cidade do Rio de Janeiro expostos a solventes. O desenho de pesquisa utilizado foi estudo transversal. Foram avaliados 55 pintores, sendo os dados sociodemográficos e da história ocupacional coletados através do Bloco de Entrevista de Saúde do Trabalhador. A faixa etária mais freqüente foi de $41-50$ anos de idade $(38,18 \%)$ e a maioria dos pintores trabalhava na universidade há mais de 10 anos (70,91\%). Quanto às condições de trabalho, $58,2 \%$ dos pintores informaram que nunca usavam equipamento de proteção individual; $52,73 \%$ desconheciam a existência da Comissão Interna de Prevenção de Acidentes de Trabalho; 92,73\% realizavam algum tipo de movimento repetitivo; $60 \%$ deles possuíam outra fonte de renda (sendo a maioria como pintor autônomo) e 87,3\% consideravam bom o relacionamento no trabalho. Vinte e cinco $(45,45 \%)$ já exerciam atividade como pintores antes de trabalharem na universidade. O inventário do local de trabalho revelou que o ambiente e as condições de trabalho eram inadequados.
\end{abstract} da Universidade Federal do Rio de Janeiro, Programa Organização do Trabalho e Saúde Mental (OTSAM).

* Trabalho baseado na Tese de Doutorado de Andréia Aparecida de Miranda Ramos, intitulada Estudo de morbidade neuropsiquiátrica em pintores dos setores de manutenção da UFRJ expostos a solventes, apresentada ao Instituto de Psiquiatria da Universidade Federal do Rio de Janeiro, em 2004.

A pesquisa recebeu auxílio do CNPq (Bolsa de Doutorado e Taxa de Bancada). Número do processo: 141800/00-3.

Contato:

Andréia Ramos

Avenida Barão de Rio Branco, 2828/904, Centro - Juiz de Fora/ MG - CEP: 36016-311

E-mail:

ramos.aam@gmail.com
Palavras-chaves: pintores, condições de trabalho, exposição ocupacional.

\section{Abstract}

This article presents the sociodemographic profile and the working conditions of wall painters exposed to solvents at a public university in Rio de Janeiro. The survey design was cross-sectional study. Fifty-five wall painters were assessed and their demographic and occupational background data were collected through interviews ("Bloco de Entrevista de Saúde do Trabalhador"). The most frequent age group was of 41-50 years old (38.18\%). Most of the painters had been working at the university for over 10 years (70.91\%). As for their working conditions, $58.2 \%$ of the painters informed that they had never worn individual protection equipment; 52.73\% were unaware of the Work Accident Prevention Committee ("Comissão Interna de Prevenção de Acidentes de Trabalho"); 92.73\% performed some kind of repetitive movement; $60 \%$ had another income source (most of them as free lancers); $87.3 \%$ ranked relationship at work as of good quality and $45.45 \%$ had already worked as wall painters before being hired at the university. Workplace inventory records have shown environmental and working conditions to be unsuitable.

Keywords: wall painters, working conditions, occupational exposure. 


\section{Introdução}

A exposição crônica a solventes está associada a queixas subjetivas relacionadas, particularmente, a funções cognitivas. Embora o exame neurológico seja, com freqüência, normal, exceto nos casos mais graves de exposição, os efeitos neuropsiquiátricos subclínicos tendem a ser mais precoces na história de exposição do indivíduo (ÖSTERBERG et al., 2000a). As anormalidades neuropsicológicas incluem disfunções comportamentais, cognitivas e emocionais (ÖSTERBERG et al., 2000b). São comumente relatadas queixas de cefaléia, tontura, fadiga, parestesias, dor e fraqueza. Queixas subjetivas de distúrbios de memória (esquecimento, dificuldade de concentração) têm sido freqüentemente alegadas (EDLING \& EKBERG, 1985; CRANMER \& GOLDBERG, 1987; WOOD \& LIOSSI, 2005; AKILA et al., 2006).

Na revisão realizada, a categoria dos pintores foi a mais referida na maioria dos estudos clínicos sobre associação entre sintomas neuropsiquiátricos e exposição ocupacional a solventes (HÄNNINEN et al., 1976; HANE et al., 1977; ARLIEN-SØBORG et al., 1979; ELOFSSON et al., 1980; HUSMAN, 1980; KURPPA \& HUSMAN, 1982; LINDSTRÖM \& WICKSTROM, 1983; CHERRY et al., 1985; LUND \& HAKANSSON, 1985; HÄNNINEN, ANTTI-POIKA \& SAVOLAINEN, 1987; GUBÉRAN et al., 1989; BRACKBILL, MAIZLISH \& FISCHBACH, 1990; CHEN et al., 1991; LUNDBERG et al., 1992; MOEN \& HOLLUND, 2000; YU et al., 2004).

Os transtornos neuropsiquiátricos relacionados a exposições a neurotoxinas estão associados a auxílios-doença, auxílios-acidente, aposentadorias por invalidez e pensões ("benefícios") concedidos pelos sistemas previdenciários de diversos países, mas nem sempre o diagnóstico que justifica o "benefício" deixa clara esta associação (CALLENDER et al., 1997; RAMOS et al., 1998; RAMOS \& SILVA FILHO, 2001, 2004). O risco relativo de pensão por incapacidade decorrente de demência pré-senil é considerado mais alto entre trabalhadores expostos a solventes do que entre grupos controle (AXELSON, HANE \& HOGSTEDT, 1976; MIKKELSEN, 1980; OLSEN \& SABROE, 1980; O'FLLYNN, MONKMAN \& WALDRON, 1987). Parece haver uma tendência em se atribuir diagnóstico de transtornos neuróticos a trabalhadores expostos a neurotoxinas considerados elegíveis para receber "benefícios". Segundo Lindström, Rihimaki e Hänninen (1984), a exposição a solventes aumenta o risco de pensões precoces por transtornos neuróticos, mas não por alcoolismo e distúrbios neuropsiquiátricos. A hipótese explicativa desses achados é que, talvez, o fato desses trabalhadores apresentarem, na maioria das vezes, queixas inespecíficas impeça a associação com a exposição ocupacional a solventes.

A avaliação do ambiente de trabalho deve ser considerada como uma etapa fundamental na avaliação de trabalhadores expostos a solventes e outros neu- rotóxicos para compreensão da relação saúde/doençatrabalho, uma vez que através dela pode-se construir inquérito do ambiente de trabalho que corrobore dados importantes evidenciados pela história ocupacional. Além disso, permite que se faça uma discussão com os trabalhadores envolvidos no processo de trabalho, suas chefias e demais setores da instituição envolvidos com saúde e segurança no trabalho com o objetivo de desenvolver propostas de educação sobre ambiente e condições de trabalho, divulgação de mais informações sobre os riscos aos quais os trabalhadores estão expostos e as medidas de proteção, individuais e coletivas, que podem ser utilizadas. Dessa forma, é possível pensar na implementação de mudanças no ambiente e na organização do trabalho que contribuam para erradicar ou minimizar os fatores de riscos identificados na avaliação do ambiente de trabalho.

O objetivo deste trabalho é descrever os achados da pesquisa referentes aos aspectos sociodemográficos do grupo de pintores estudados e suas condições de trabalho. Os demais resultados da pesquisa serão apresentados em outro artigo.

\section{Metodologia}

A universidade conta com o número de 80 pintores em seu quadro funcional distribuídos em 31 diferentes unidades da instituição, de acordo com os dados da Sub-Reitoria de Pessoal e Serviços Gerais (SR-4) e da Divisão de Saúde dos Trabalhadores (DVST).

Antes de se iniciar o estudo, a pesquisadora responsável realizou contato pessoal com os chefes dos setores de manutenção para esclarecer os objetivos da pesquisa bem como sensibilizar para a participação na mesma. Os indivíduos que participaram da pesquisa foram convocados por meio de telegrama, enviado pela DVST, para realização do Exame Periódico de Saúde, no qual estava inserida a presente pesquisa. Foram realizadas até 4 convocações - via telegrama e telefone - durante período de 1 ano (2003-2004).

Foi realizado um estudo de corte transversal.

\section{Local do estudo}

Os trabalhadores foram avaliados no prédio da DVST, localizado em um dos campi da universidade. O horário foi agendado previamente com os responsáveis técnicos pelos setores de manutenção de forma a obedecer ao cronograma de atividades dos pesquisados, evitando interferência na rotina de trabalho.

\section{Amostra}

A amostra foi composta de 55 trabalhadores. Ao chegarem à DVST, os funcionários eram encaminhados para exame clínico e coleta de sangue para realização de exames laboratoriais. Após isso, a pesquisa- 
dora conversava com eles sobre o objetivo do estudo e esclarecia que a participação era facultativa. Após aceitação em participar, era entregue o Termo de Consentimento Livre e Esclarecido e se iniciava entrevista ocupacional e avaliação psiquiátrica, com a aplicação do Bloco de Entrevista em Saúde Mental do Trabalhador - Best Doc I e II (Anexos 1 e 2), da entrevista psiquiátrica semi-estruturada Clinical Interview Schedule (1970) - CIS (GOLDBERG et al., 1970; BLAY et al., 1991) e do questionário de rastreamento de sintomas neuropsiquiátricos em indivíduos expostos a solventes - Q16. A entrevista durava, em média 60 a 90 minutos. Terminada esta avaliação, os trabalhadores eram agendados para avaliação neuropsicológica e realização de eletroencefalograma (EEG) em um dos institutos da universidade, localizado em outro campus. O resultado da avaliação clínico-neuropsiquiátrica será objeto de outro artigo.

Os pintores que, no momento da pesquisa, estavam afastados de sua função por licença médica ou desviados de função foram convocados com o objetivo de tentar identificar se uma possível causa do afastamento foi a presença de sintomas relacionados à exposição. Considerou-se importante a avaliação destes trabalhadores para melhor caracterização da população estudada, uma vez que estudos realizados apontam como freqüente motivo de afastamento de função a presença de sintomas neuropsiquiátricos que não são relacionados à exposição durante o exame periódico de saúde e que, posteriormente, análises mais pormenorizadas identificam a associação entre os sintomas e a exposição ocupacional ao solvente (CHERRY, VENABLES \& WALDRON, 1984; BRACKBILL, MAIZLISH \& FISCHBACH, 1990). O levantamento dos indivíduos afastados por licença médica ou desviados de função foi feito a partir de dados da SR-4 e da DVST, ambas instâncias administrativas que controlam as licenças e os afastamentos dos trabalhadores da instituição. Os dados sociodemográficos foram obtidos utilizando o Best Doc I.

\section{Inventário do local de trabalho}

Para avaliação das condições de trabalho, realizou-se inventário do local de trabalho. A universidade possuía, no momento da pesquisa, 31 setores de manutenção distribuídos em 31 diferentes unidades, onde se localizavam várias oficinas, entre elas a de serviço de pintura.

Realizou-se visita ao local de trabalho para estudar o processo de trabalho, verificando o fluxograma e a jornada de trabalho, as avaliações ambientais e médicas feitas pela empresa, a relação de trabalhadores e suas respectivas funções e as plantas da empresa. Com isso, construiu-se o inventário do ambiente de trabalho de uma das oficinas de pintura do setor de manutenção da universidade, escolhida pelo critério de sua localização e pela maior facilidade de acesso em virtude da proximidade com o local onde estava se desenvolvendo a pesquisa.

A avaliação do ambiente de trabalho foi realizada pela pesquisadora responsável pelo estudo, que colheu os dados referentes à organização do trabalho. Uma equipe de pesquisadores do CESTEH/Fiocruz, do Laboratório de Pesquisa em Solventes, composta por dois químicos e dois técnicos em laboratório, foi a responsável pela coleta dos dados da avaliação ambiental (medida da exposição ambiental, medida biológica, registro fotográfico dos locais de trabalho - oficina de pintura e local de realização do trabalho). A equipe de pesquisadores foi acompanhada por um técnico em segurança do trabalho da DVST.

Antes de iniciar o estudo do ambiente de trabalho, foi enviado ofício ao chefe do setor de manutenção comunicando a ida dos pesquisadores e solicitando aprovação da atividade. Este comunicado foi enviado pela diretora da DVST e continha os esclarecimentos necessários a respeito da pesquisa em andamento.

Para análise dos dados, utilizou-se o programa de análises estatísticas SPSS versão 8. Utilizou-se a técnica de estatística descritiva, de análise exploratória de dados.

\section{Resultados}

Características sociodemográficas, da história ocupacional e da história de saúde do grupo de 55 pintores dos setores de manutenção da universidade

O fluxograma da Figura 1 apresenta como se deu a definição do grupo estudado.

Dos trabalhadores que não compareceram $(n=25)$ às convocações (Figura 1), cabe ressaltar:

- Do trabalhador falecido: em seu prontuário, consta diagnóstico de hepatopatia como causa de morte, tendo sido questionado a presença do fator de exposição ocupacional a solventes associado à doença hepática. Porém, não há nenhum registro de que esta hipótese tenha sido investigada.

- Dos trabalhadores licenciados: um estava afastado por apresentar diagnóstico de adenocarcinoma de próstata e estar em tratamento oncológico; o segundo estava licenciado para tratamento de etilismo; e o terceiro por apresentar doença pulmonar obstrutiva crônica.

Todos os trabalhadores que participaram do estudo (Figura 1) são do sexo masculino. A faixa etária mais freqüente foi de 41-50 anos de idade (38,18\%). O estado civil mais freqüente foi o de casado $(81,82 \%)$. Quanto à naturalidade, 58,18\% são procedentes do município do Rio de Janeiro, seguidos pela Região Nordeste (29,09\%). Os pintores pesquisados possuíam 
baixa escolaridade: $69,09 \%$ com $1^{\circ}$ grau incompleto (Tabela 1).

A maioria dos pintores pesquisados despendia um tempo significativo para se deslocar de sua residência até o local de trabalho: $32,73 \%$ gastavam de $1 \mathrm{~h}$ a $1 \mathrm{~h} 30 \mathrm{~m}$ e $29,09 \%$ gastavam entre $1 \mathrm{~h} 30$ e $2 \mathrm{~h}$ para efetuar este deslocamento (Tabela 2). A maioria dos pintores estava na universidade há 10-15 anos (70,91\%) (Tabela 2).

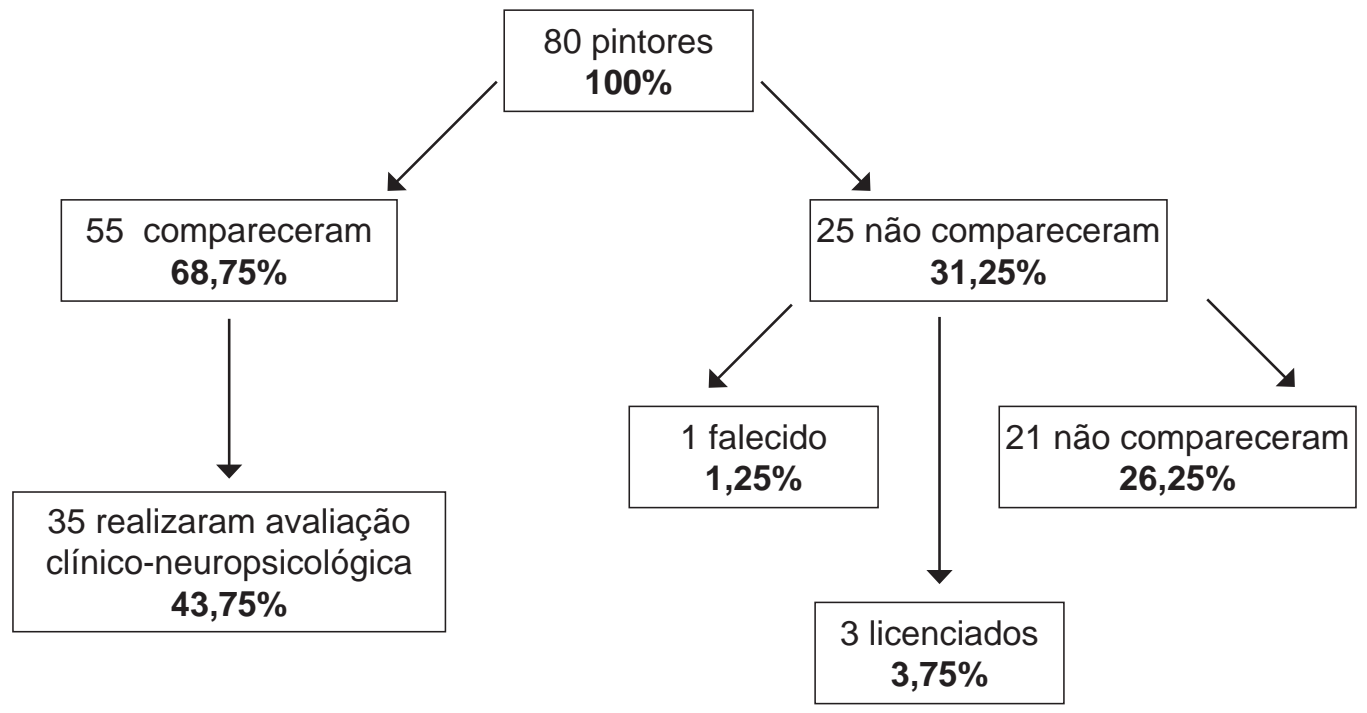

Figura 1 População global de pintores da universidade e a amostra que participou do estudo

Tabela 1 Distribuição das características sociodemográficas de uma amostra de 55 pintores de setores de manutenção de uma universidade pública do Rio de Janeiro, abril/2003 a março/2004

\begin{tabular}{|c|c|c|}
\hline Características & $N$ & $\%$ \\
\hline \multicolumn{3}{|l|}{ Gênero } \\
\hline Feminino & 0 & 0,00 \\
\hline Masculino & 55 & 100,00 \\
\hline \multicolumn{3}{|l|}{ Faixa etária (anos) } \\
\hline $31-40$ & 14 & 25,46 \\
\hline $41-50$ & 21 & 38,18 \\
\hline $51-60$ & 17 & 30,90 \\
\hline$\geq 61$ & 3 & 5,46 \\
\hline \multicolumn{3}{|l|}{ Estado civil } \\
\hline Casado & 45 & 81,82 \\
\hline Solteiro & 6 & 10,91 \\
\hline Separado & 4 & 7,27 \\
\hline \multicolumn{3}{|l|}{ Naturalidade } \\
\hline Rio de Janeiro (cidade) & 32 & 58,18 \\
\hline Rio de Janeiro (estado) & 4 & 7,27 \\
\hline Outros estados do Sudeste & 3 & 5,46 \\
\hline Nordeste & 16 & 29,09 \\
\hline \multicolumn{3}{|l|}{ Escolaridade } \\
\hline Analfabeto & 1 & 1,80 \\
\hline $1^{\circ} \mathrm{Grau}$ incompleto & 38 & 69,10 \\
\hline $1^{\circ}$ Grau completo & 9 & 16,37 \\
\hline $2^{\circ}$ Grau incompleto & 4 & 7,27 \\
\hline $2^{\circ}$ Grau completo & 3 & 5,46 \\
\hline
\end{tabular}


Tabela 2 Características do trabalho atual de uma amostra de 55 pintores de setores de manutenção de uma universidade pública do Rio de Janeiro, abril/2003 a março/2004

\begin{tabular}{|c|c|c|}
\hline Características & $N$ & $\%$ \\
\hline \multicolumn{3}{|c|}{ Tempo de deslocamento para o trabalho (minutos) } \\
\hline $15-30$ & 4 & 7,27 \\
\hline $31-60$ & 13 & 23,64 \\
\hline $61-90$ & 18 & 32,73 \\
\hline $91-120$ & 16 & 29,09 \\
\hline$\geq 121$ & 4 & 7,27 \\
\hline \multicolumn{3}{|l|}{ Tempo na empresa (anos) } \\
\hline $10-15$ & 39 & 70,91 \\
\hline $16-20$ & 12 & 22,82 \\
\hline $21-25$ & 4 & 7,27 \\
\hline \multicolumn{3}{|l|}{ Função exercida } \\
\hline Função real de pintor & 43 & 78,18 \\
\hline Desvio de função & 12 & 21,82 \\
\hline \multicolumn{3}{|l|}{ Tempo na função atual (anos) } \\
\hline$<1$ & 2 & 3,64 \\
\hline $1-10$ & 8 & 14,55 \\
\hline $11-20$ & 41 & 74,54 \\
\hline$\geq 21$ & 4 & 7,27 \\
\hline \multicolumn{3}{|l|}{ Horas extras } \\
\hline Sim & 8 & 14,55 \\
\hline Não & 47 & 85,45 \\
\hline \multicolumn{3}{|l|}{ Tempo de jornada } \\
\hline Normal (8 horas diárias) & 53 & 96,36 \\
\hline Variável (em número de horas diárias) & 2 & 3,64 \\
\hline
\end{tabular}

Doze $(21,82 \%)$ estavam desviados de função, ocupando diferentes funções e por tempo também diferentes. Dez deles estavam exercendo funções fora do setor de manutenção (almoxarifado, portaria, farmácia), enquanto dois continuavam em setores de manutenção: um na oficina de refrigeração e o outro na oficina de pintura com função administrativa. Nove desses trabalhadores foram desviados da função de pintor por ter sido considerado que suas queixas clínicas guardavam relação com a exposição a solventes: seis com sinais e sintomas sugestivos de rinite alérgica, dois com queixa de cefaléia intensa quando em contato com tintas e outros produtos químicos e um com queixa de sintomas neuropsiquiátricos. Este último teve a associação entre as queixas neuropsiquiátricas e a exposição a solventes estabelecida pelo psiquiatra que o assiste. Os outros três pintores desviados de função não tiveram suas queixas associadas à exposição a solventes: um apresentava queixa de pa- restesia em membros inferiores, um apresentava queixas neuropsiquiátricas e um, queixa de diminuição da acuidade visual à esquerda (Tabela 2).

A maioria $(85,45 \%)$ dos pesquisados não fazia hora extra na empresa e o tipo de jornada era quase exclusivamente de 8 horas diária. Os dois $(3,64 \%)$ trabalhadores que responderam ter jornada de trabalho variável explicaram que esse fato ocorre porque as unidades onde trabalhavam tinham um grande fluxo de atividades durante o período diurno, o que dificultava a execução de pintura. Assim, era necessário cumprirem a tarefa fora do horário de funcionamento da unidade. No entanto, essa variação não era permanente (Tabela 2).

Quanto às condições de trabalho (Tabela 3), 58,2\% dos pintores informaram que nunca usavam qualquer equipamento de proteção individual (EPI); 52,73\% desconheciam a existência da Comissão Interna de 
Prevenção de Acidentes de Trabalho (CIPA); 92,73\% realizavam algum tipo de movimento repetitivo; 83,63\% trabalhavam em posição alternada (andando, em pé); $60 \%$ deles possuíam outra fonte de renda (destes, apenas dois deles informaram ter outra fonte de renda exercendo outra função que não a de pintor); e $87,3 \%$ consideravam bom o relacionamento no trabalho (com colegas e com a chefia).

A história de trabalho revelou que 58,18\% dos pintores avaliados começaram a trabalhar com idade entre 11 e 15 anos; 63,64\% e 72,73\% nunca sofreram acidente de trabalho e doença ocupacional, respectivamente, ao longo de suas vidas laborativas; 58,19\% não haviam realizado exame periódico de saúde anteriormente na universidade (Tabela 4).
Um outro dado importante é que 25 (45,45\%) dos 55 pintores já trabalhavam nessa profissão antes de ingressarem na universidade. Quando se avaliou o tempo de exposição desses pintores, utilizando o critério anos como pintor, verificou-se que a freqüência maior foi entre o período de 21 a 30 anos de exercício da função $(45,7 \%)$.

A história de saúde destacou que 16 (29,10\%) dos 55 pintores avaliados informaram uso de algum tipo de medicamento no momento da realização do estudo. Os medicamentos citados foram: analgésicos, antiinflamatórios não hormonais, anti-hipertensivos, broncodilatadores, antiácidos e descongestionante nasal. Um indivíduo relatou uso esporádico de benzodiazepínico (clonazepam até $0,5 \mathrm{mg}$ ) prescrito pelo seu médico.

Tabela 3 Características das condições de trabalho de uma amostra de 55 pintores de setores de manutenção de uma universidade pública do Rio de Janeiro, abril/2003 a março/2004

\begin{tabular}{|c|c|c|}
\hline & $N$ & $\%$ \\
\hline \multicolumn{3}{|c|}{ Uso de EPI (Equipamento de Proteção Individual) } \\
\hline Às vezes & 14 & 25,45 \\
\hline Sempre & 9 & 16,36 \\
\hline Nunca & 32 & 58,19 \\
\hline \multicolumn{3}{|c|}{ CIPA (Comissão Interna de Prevenção de Acidentes) no local de trabalho } \\
\hline Sim & 4 & 7,27 \\
\hline Não & 22 & 40,00 \\
\hline Não sabe & 29 & 52,73 \\
\hline \multicolumn{3}{|c|}{ Outras fontes de renda } \\
\hline Sim & 33 & 60,00 \\
\hline Não & 22 & 40,00 \\
\hline \multicolumn{3}{|c|}{ Local de refeição } \\
\hline Sim & 29 & 52,73 \\
\hline Não & 26 & 47,27 \\
\hline \multicolumn{3}{|c|}{ Movimentos repetitivos } \\
\hline Sim & 51 & 92,73 \\
\hline Não & 4 & 7,27 \\
\hline \multicolumn{3}{|c|}{ Posição no trabalho } \\
\hline Sentado & 2 & 3,64 \\
\hline Em pé & 6 & 10,91 \\
\hline Andando & 1 & 1,82 \\
\hline Alternado & 46 & 83,63 \\
\hline \multicolumn{3}{|c|}{ Relacionamento no trabalho } \\
\hline Bom & 48 & 87,27 \\
\hline Razoável & 7 & 12,73 \\
\hline Ruim & 0 & 0,00 \\
\hline
\end{tabular}


Tabela 4 História de trabalho de uma amostra de 55 pintores de setores de manutenção de uma universidade pública do Rio de Janeiro, abril/2003 a março/2004

\begin{tabular}{lcc}
\hline \multicolumn{1}{c}{ Características } & $N$ & $\%$ \\
\hline $\begin{array}{l}\text { Idade em que começou a trabalhar } \\
\leq 10\end{array}$ & 9 & 16,36 \\
$11-15$ & 12 & 58,18 \\
$16-19$ & 2 & 21,82 \\
$\geq 20$ & & 3,64 \\
História de acidentes de trabalho & 20 & 36,36 \\
Sim & 35 & 63,64 \\
Não & & \\
História de doenças ocupacionais & 15 & 27,27 \\
Sim & 40 & 72,73 \\
Não & & \\
Exame periódico anterior & & 41,81 \\
Sim & 23 & 58,19 \\
Não & 32 & \\
\hline
\end{tabular}

Nove $(16,4 \%)$ dos 55 pintores informaram que faziam ou já haviam feito tratamento psiquiátrico e/ou psicológico: 4 fizeram tratamento psiquiátrico por apresentar diagnóstico de etilismo; 2 fizeram tratamento psicoterápico por apresentar sintomas ansiosos; 1 fez tratamento para depressão em 1999; 1 faz acompanhamento psicoterápico por apresentar diagnóstico de etilismo; 1 fazia acompanhamento psiquiátrico por apresentar diagnóstico de transtorno mental relacionado à exposição crônica a solventes. Três trabalhadores com história de tratamento psiquiátrico e/ ou psicológico foram considerados suspeitos de apresentar sintomas neuropsiquiátricos pelos critérios da avaliação psiquiátrica e do questionário Q16 (resultados apresentados em outro trabalho). Um dos pintores foi encaminhado para o tratamento psiquiátrico por apresentar suspeita de morbidade neuropsiquiátrica associada à exposição ocupacional a solventes. Este último trabalhador foi o único da amostra que teve a associação estabelecida por profissionais da saúde mental do trabalhador.

Vinte e quatro $(43,64 \%)$ dos 55 pintores apresentaram história patológica pregressa positiva: 8 com história de doença pulmonar, 7 de hipertensão arterial, 3 de doença gastrointestinal, 2 de doença osteoarticular, 3 de rinite alérgica e 1 de traumatismo cranioencefálico.

\section{Resultados do inventário do local de trabalho}

O fato de os setores de manutenção estarem localizados em diferentes locais dificultou a realização da avaliação ambiental, prejudicando o levantamento dos dados referentes à intensidade da exposição (ní- veis de exposição biológica e ambiental). Portanto, não foi possível avaliar todas as oficinas, tendo-se optado pela descrição de uma oficina de pintura localizada em um dos campi. As greves também foram fatores determinantes para o não cumprimento dos objetivos iniciais das avaliações ambiental e biológica, pois era necessário que os trabalhadores estivessem em atividade diária para avaliação dos níveis de exposição.

Apenas uma unidade da universidade foi avaliada durante a jornada de trabalho dos pintores. Situava-se em um dos prédios onde funcionam salas de aula, laboratórios de pesquisa e setores administrativos. No entanto, sua localização é no subsolo deste prédio, onde também se encontram algumas salas de aula, laboratórios e as demais oficinas daquele setor de manutenção.

Para se ter acesso à oficina de pintura, era necessário descer dois lances de escadas e caminhar cerca de $100 \mathrm{~m}$ pelo corredor do subsolo. Em frente à oficina, havia uma porta que dava acesso a um pátio, mas sem saída para a área superior, de forma que todo o material usado em alguma tarefa fora do subsolo tinha que ser transportada pelas escadas, o que significa, muitas vezes, carregar peso por uma distância maior, pois se tratava de um prédio de grandes dimensões. Todo o material utilizado durante a jornada de trabalho era carregado pelos próprios pintores, não havendo um instrumento próprio para isso (como, por exemplo, um carrinho de mão).

Ao lado da oficina de pintura localizava-se a oficina de marcenaria, onde se observou ruído produzido pelos maquinários. 
No momento da avaliação da oficina, apenas três pintores estavam trabalhando. Eles informaram que dois outros pintores desse setor estavam prestando serviços para outros setores de manutenção.

A seguir é apresentada descrição do ambiente de trabalho.

Descrição da sala e a ante-sala, respectivamente, da oficina onde os trabalhadores guardavam os materiais de trabalho (ferramentas, tintas), determinados alimentos que consumiam (café, açúcar, água etc.) e seus pertences pessoais, onde realizavam e preparavam algumas refeições (café-da-manhã, café-da-tarde) e onde descansavam durante o período de pausa: tratava-se de uma pequena sala, sem janela, com um aparelho de ar condicionado, um sofá, uma mesa e dois armários onde eram guardados os pertences pessoais e os materiais de trabalho. $\mathrm{Na}$ ante-sala havia uma pia e uma pequena prateleira. Pôde-se observar que não existia um local separado para os materiais de trabalho, os pertences pessoais e os alimentos. Os trabalhadores informaram que, geralmente, almoçavam em algum restaurante próximo ao prédio, pois não existia refeitório nesta unidade para os funcionários.

Mesmo durante o dia era necessário manter as luzes da oficina acessas em virtude da escassez de luz natural no ambiente.

Foi observada a proximidade dos materiais utilizados na execução das tarefas de trabalho - inclusive as tintas - com os utensílios de cozinha, usados na preparação de alimentos. Neste mesmo local, localizavase a prateleira usada para colocar algumas ferramentas de trabalho e a água consumida pelos trabalhadores. Também se pôde verificar que algumas latas de tintas e outros materiais cuja composição contém solventes estavam abertas. Os pintores explicaram que as tintas e as demais substâncias em uso ficavam armazenadas na oficina até acabar o consumo. A maior parte das tintas utilizadas nesta oficina era composta por $10 \%$ a $25 \%$ de solventes (terebintina, água-raz, álcool etílico), $25 \%$ a $65 \%$ de não voláteis (óleos vegetais ou resinados de metais pesados - chumbo, manganês e cobalto), $25 \%$ a $50 \%$ de pigmentos (óxido de zinco, óxido de titânio, óxido de cromo, óxido de ferro, cromato de chumbo, cádmio e outros metais).

Terminada a visita na oficina de pintura, a equipe de pesquisadores seguiu, com os pintores, para o local onde iria se realizar o trabalho daquele dia: a pintura de portas de um dos banheiros localizados no primeiro andar do prédio. Neste dia, os pintores precisaram carregar até o local da pintura duas latas de tinta, pincéis, pequenos rolos e lixas. Como não havia outro serviço escalado para aquela manhã, os três pintores trabalharam juntos (cabe esclarecer que a universidade vivia um momento de paralisações e greves dos funcionários, de forma que poucas unidades estavam funcionando plenamente em algumas etapas da pesquisa). No local da pintura foram instalados dois pontos para medir o nível de solvente no ambiente.
Observou-se que nenhum dos trabalhadores usava qualquer tipo de equipamento de proteção individual durante toda a execução do trabalho e que usavam uniforme fornecido pela instituição. Os trabalhadores informaram que, após a jornada de trabalho, eles tomavam banho em um banheiro localizado fora da oficina de pintura e trocavam de roupa. Pôde-se também observar questões ergonômicas: a posição em que ficavam os pintores no momento de realizar as atividades e a forma como manuseavam as ferramentas - a presença de movimentos repetitivos.

\section{Discussão}

O estudo realizado identificou a escassez de informação dos trabalhadores quanto à presença de CIPAs nos locais de trabalho, evidenciando uma participação pouco efetiva nas discussões acerca da organização e das condições do ambiente de trabalho.

Outro dado importante é a alta freqüência de trabalhadores que nunca haviam se submetido a exames periódicos anteriormente, considerando-se o longo período que eles já trabalhavam na instituição (o menor tempo no serviço foi de 10 anos). Esse dado reflete a dificuldade em se estabelecer parâmetros mais apurados sobre esta população de trabalhadores no que diz respeito à avaliação neuropsiquiátrica, pois não se dispõe de dados anteriores para se comparar com os atuais. Essa limitação tem sido apontada por outros estudos que discutem a questão do nexo entre exposição ocupacional a solventes e desenvolvimento de sintomas neuropsiquiátricos (CHERRY, VENABLES \& WALDRON, 1984; HARTMAN, 1988; BAKER, 1988; FELDMAN, 1999; VAN DER HOEK, VERBERK \& HAGEMAN, 2000; FERIGOLO et al., 2000; TRIEBIG \& HALLERMANN, 2001; GLINA, ROCHA \& MENDONÇA, 2001).

A investigação dessa amostra de trabalhadores corrobora a discussão de que populações de pintores são expostas a diferentes agentes - físicos, químicos, ergonômicos - no ambiente de trabalho (SINGER, 1990; ZAVARIZ \& GLINA, 1992; ORELLANA \& SALLATO, 1995).

Estudos sobre ambientes de trabalho relatam que, em indústrias, podem ser identificados até nove agentes nocivos simultaneamente (RENTZSCH, PRESCHER \& TOLKSDORF, 1992). Estudos sobre os efeitos combinados a agentes ambientais no trabalho, embora complexos, constituem um importante desafio na área da saúde do trabalhador, especificamente no campo da saúde mental e trabalho (LACAZ, 1997). No entanto, tem se verificado um aumento de publicações sobre os efeitos combinados, indicando um crescente interesse por uma abordagem menos restritiva para as explicações de nexos associativos, especialmente sobre a exposição a agentes químicos e ruído (NIKOLOV, 1974; HUMES, 1984; BOETTCHER et al., 1987; 
MANNINEN, 1993; MEHNERT, GRIEFAHN \& BRODE, 1993; CROFTON, LASSITER \& REBERT, 1994; SASSKORTSAK, COREY \& ROBERTSON, 1995; BESS \& HUMES, 1998; MORATA \& LEMASTERS, 2001). Isso nos leva a pensar num processo de adoecimento contínuo, em que diversas variáveis devem ser analisadas. No presente estudo, destacou-se a exposição a outros agentes, como ruído, agentes biológicos (nas unidades que possuem laboratórios), movimentos repetitivos (lixar, manusear rolo de tinta e pincel), carregar peso, posições inadequadas na realização das atividades, outros agentes químicos.

Tanto o dado de ter outra fonte de renda (exercendo a função de pintor de modo informal) quanto o de já ter a profissão de pintor antes de ingressar na universidade podem ser considerados como indicadores de tempo de exposição (duração) e corroborar a suspeita de que, geralmente, pintores possuem dupla - e por que não dizer múltipla - exposição ocupacional a solventes. Infelizmente, esses dados não permitem verificar a intensidade de tais exposições uma vez que não se dispõe de informações de avaliações biológicas anteriores, mas constituem dados fundamentais para a composição da associação entre possíveis queixas clínicas e exposição a solventes.

A alta freqüência de história negativa de acidentes de trabalho e de doença relacionada ao trabalho numa amostra em que se esperava resultado contrário - por se tratar de uma função sujeita a acidentes do tipo cortes, quedas e doenças como dermatites, doenças pulmonares - pode tentar ser explicada pela baixa percepção que os trabalhadores avaliados no estudo tinham dos riscos de seu trabalho e pelo desconhecimento dos possíveis efeitos dos agentes aos quais estão expostos no ambiente laborativo.

O dado de que apenas um dos trabalhadores pesquisados foi encaminhado a um serviço de saúde mental com suspeita de sintomas psiquiátricos relacionados à exposição ocupacional a solventes remete à discussão acerca do baixo índice de identificação de possíveis sintomas neuropsiquiátricos relacionados a este tipo de exposição em serviços básicos de saúde. Mas a dificuldade de se construir esse nexo parece se estender aos serviços de saúde de atenção terciária, onde se verifica, também, baixa freqüência de diagnósticos neuropsiquiátricos relacionados ao trabalho (MARI, 1987; PENAYO, KULLGREN \& CALDERA, 1990; EL-RUFAIE \& ABSOOD, 1993).

A variável ter feito ou fazer tratamento psiquiátrico e/ou psicológico, quando correlacionada com a avaliação psiquiátrica e o questionário Q16, não mostrou significância estatística entre os indivíduos considerados suspeitos. Assim como ter história patológica pregressa positiva também não demonstrou significância estatística quando essa variável foi correlacionada com a avaliação psiquiátrica. No entanto, é importante ressaltar que a presença de uma outra doença não exclui a possibilidade da existência de sintomas neuropsiquiátricos. Esse dado faz emergir a discussão de se excluir ou não trabalhadores com outras doenças de estudos sobre associação entre exposição ocupacional a solventes e desenvolvimento de sintomas neuropsiquiátricos. Talvez a melhor forma de se controlar essa variável seja a utilização de grupo-controle.

A análise de variáveis independentes evidenciou associação entre algumas delas e os critérios diagnósticos da OMS e o resultado do questionário Q16. Verificou-se que foram as variáveis 'uso de equipamento de proteção individual' e história de ter 'outra fonte de renda' as que tiveram significância estatística. A condição histórica de já ser pintor antes de trabalhar na universidade foi considerada, neste estudo, um indicador do tempo de exposição. Essas variáveis podem, portanto, ser consideradas com indicadores de uma possível associação. O tamanho da amostra pode explicar a dificuldade para se estabelecer a significância estatística.

A discussão que se faz ao avaliar essas duas variáveis é sobre a importância da significância estatística e da relevância clínica na análise dos dados deste estudo. O conceito de significância clínica "se refere a um resultado cuja magnitude seja de tal forma expressiva que mereça ser considerada pelo pesquisador" (LUIZ \& MAGNANINI, 2002, p. 304). Dessa forma, "estudos estatisticamente 'não significativos' também são importantes de ser relatados, seja por razões de estudos futuros ou sistematizações, seja pela eventual presença de significância clínica no estudo" (LUIZ \& MAGNANINI, 2002, p. 304). Considera-se que os resultados do presente trabalho possam ser avaliados, especialmente aqueles relacionados às variáveis uso de EPI e condição de já trabalhar como pintor antes de ingressar na universidade, a partir de sua significância clínica.

As variáveis escolaridade, idade, tempo na função e uso de bebida alcoólica não apresentaram associação positiva. Esses resultados se mantiveram também no grupo de 35 pintores que se submeteram à avaliação psicológica.

Os resultados deste estudo representam uma análise exploratória e descritiva, devendo ser consideradas suas limitações e implicações. Deve-se, portanto, tomá-los como proposta de criar subsídios para futuros estudos que possam explorar a hipótese de nexo causal entre exposição ocupacional a solventes em pintores dos setores de manutenção da universidade pesquisada. Uma vez explicitado esse caráter do estudo, sabe-se que a extrapolação dos dados encontrados nesta pesquisa limita-se à população de pintores da instituição analisada. No entanto, acredita-se que seja importante a realização de tais estudos com o objetivo de contribuir com subsídios para políticas de melhoria nos ambientes de trabalho e para criação de bancos de dados que permitam a comparação entre estudos. 
Dentre as políticas de melhoria no ambiente de trabalho, destaca-se a importância da realização de uma ampla discussão com a população de pintores pesquisados, suas chefias e setores da instituição envolvidos com saúde e segurança no trabalho com o

\section{Referências}

ARLIEN-SØBORG, P. et al. Chronic painters' syndrome. Acta Neurol Scand., n. 60, p. 149-156, 1979.

AKILA, R. et al. Memory performance profile in occupational chronic solvent encephalopathy suggests working memory dysfunction. J. Clin. Exp. Neuropsychol., v. 28, n. 8, p. 1307-1326, 2006.

AXELSON, O.; HANE, H.; HOGSTEDT, C. A casereferent study on neuropsychiatric disorders among workers exposed to solvents. Scand. J. Work Environ. Health, n. 2, p. 14-20, 1976.

BAKER JR., E. L. Neurologic and behavioral disorders. In: LEVY, B. S.; WEGMAN, D. H. Occupational Health: recognizing and preventing work-related disease. Boston/Toronto: Little, Brown and Company, 1988. p. 399-412.

BESS, F. H.; HUMES, L. E. Fundamentos da audiologia. 2. ed. Porto Alegre: ArtMed, 1998.

BLAY, S. L. et al. The use of the clinical interview schedule for the evaluation of mental health in the aged community. Psychol. Med., n. 22, p. 525-530, 1991.

BOETTCHER, F. A. et al. Synergistic interaction of noise and other ototraumat agents. Ear Hear, n. 8, p. 192-212, 1987.

BRACKBILL, R. M.; MAIZLISH, N.; FISCHBACH, N. Risk of neuropsychiatric disability among painters in the United States. Scand. J. Work Environ. Health, n. 16, p. 182-188, 1990.

CALLENDER, T. J. et al. Social and economic impact of neurotoxicity in hazardous waste workers in Lenoir, North Carolina. Environ. Research, n. 73, p. 166-174, 1997.

CHEN, J. D. et al. Exposure to mixtures of solvents among paint workers and biochemical alterations of liver function. B. J. Ind. Med., n. 48, p. 696-701, 1991.

CHERRY, N. et al. Neurobehavioural effects of repeated occupational exposure to toluene and paint solvents. B. J. Ind. Med., n. 42, p. 291-300, 1985.

CHERRY, N.; VENABLES, H.; WALDRON, H. A. British studies on the neuropsychological effects of solvent exposure. Scand. J. Work Environ. Health, n. 10, supl. 11, p. 10-12, 1984.

CLINICAL INTERVIEW SCHEDULE. A manual for use in conjunction with the general practice research unit's standardised psychiatric interview. London: Institute of Psychiatry, 1970. objetivo de desenvolver propostas de educação sobre ambiente e condições de trabalho, divulgação de mais informações sobre os riscos aos quais os pintores estão expostos e as medidas de proteção, individuais e coletivas, que podem ser utilizadas.

CRANMER, J. M.; GOLDBERG, L. Proceeding of the work-shop on neuro-behavioral effects of solvents. Neurotoxicology, n. 7, p. 1-95, 1987.

CROFTON, K. M.; LASSITER, T. L.; REBERT, C. S. Solvent-induced ototoxicity in rats: na atypical selective mid-freqüency hearing deficit. Hear Res., n. 80, p. 25-30, 1994.

EDLING, C.; EKBERG, K. No acute behavioral effects of exposure to styrene: a safe level of exposure? B. J. Ind. Med., n. 42, p. 301-304, 1985.

ELOFSSON, S. et al. Exposure organic solvents: a cross-sectional epidemiologic investigation on occupationally exposed car and industrial spray painters with special reference to the nervous system. Scand. J. Work Environ. Health, n. 6, p. 239273, 1980.

EL-RUFAIE, O. E. F; ABSOOD, G. H. Minor psychiatric morbidity in primary health care: prevalence, nature and severity. The Int. J Social. Psych., v. 3, n. 39, p. 159-166, 1993.

FELDMAN, R. G. Occupational and environmental neurotoxicology. Philadelphia: Lippincott-Raven, 1999.

FERIGOLO, M. et al. Aspectos clínicos e farmacológicos do uso de solventes. J. Bras. Psiquiatria, n. 9, p. 331-341, 2000.

GLINA, D. M. R.; ROCHA, L. E.; MENDONÇA, M. G. V. Saúde mental e trabalho: uma reflexão sobre o nexo com o trabalho e o diagnóstico, com base na prática. Cad. Saúde Pública, n. 17, v. 3, p. 607-616, 2001.

GOLDBERG, D. P. et al. A Standardized psychiatric interview for use in community surveys. British J. Prev. Soc. Med., n. 24, p. 18-23, 1970.

GUBÉRAN, E. et al. Disability, mortality, and incidence of cancer among Geneva painters and electricians: a historical prospective study. Br. J. Ind. Med., n. 46, p. 16-23, 1989.

HANE, M. et al. Psychological function changes among house painters. Scand. J. Work Environ. Health, n. 3, p. 91-99, 1977.

HÄNNINEN, H. et al. Behavioral effects of long-term exposure to a mixture of organic solvents. Scand. J. Work Environ. Health, n. 4, p. 240-255, 1976.

HÄNNINEN, H.; ANTTI-POIKA, M.; SAVOLAINEN, P. Psychological performance, toluene exposure and alcohol consumption in rotogravure printers. Int. Arch. Occup. Environ. Health, n. 59, p. 475-483, 1987. 
HARTMAN, D. E. Neuropsychological toxicology: identification and assessment of human neurotoxic syndromes. New York: Perganon Press, 1988.

HUMES, L. E. Noise-induced hearing loss a influenced by other agents and by some physical characteristics individual. J. Acoustic Soc. Am., n. 76, p. 1318-1329, 1984.

HUSMAN, K. Symptoms of car painters with lonfterm exposure to a mixture of organic solvents. Scand. J. Work Environ. Health, n. 6, p. 19-32, 1980.

KURPPA, K.; HUSMAN, K. Car painters' exposure to a mixture of organic solvents. Scand. J. Work Environ. Health, n. 8, p. 137-140, 1982.

LACAZ, F. A. C. Saúde dos trabalhadores: cenários e desafios. Cad. Saúde Pública, n. 13, supl. 2, p. 7-19, 1997.

LINDSTRÖM, K.; RIHIMAKI, H.; HÄNNINEN, H. Occupational solvent exposure and neuropsychiatric disorders. Scand. J. Work Environ. Health, n. 10, p. 321-323, 1984.

LINDSTRÖM, K.; WICKSTRÖM, G. Psychological function changes among maintenance house painters exposed to low levels of organic solvent mixtures. Acta Psych. Scand., n. 67, supl. 303, p. 81-91, 1983.

LUIZ, R. B.; MAGNANINI, M. O tamanho de amostra em investigações epidemiológicas. In: MEDRONHO, R. A. et al. (Eds.). Epidemiologia. São Paulo: Atheneu, 2002. p. 295-307.

LUND, I.; HAKANSSON, M. Normal serum activities of liver enzymes in Swedish paint industry workers with heavy exposure to organic solvents. Brit. J. Ind. Med., n. 42, p. 596-600, 1985.

LUNDBERG, I. et al. Diagnoses of alcohol abuse and other neuropsychiatric disorders among house painters compared with house carpenters. Brit. J. Ind. Med., n. 49, p. 409-415, 1992.

MANNINEN, O. Synopsis of studies on combined effects. In: CONGRESS NOISE AS A PUBLIC HEALTH PROBLEM, 6., 1993, Nice. Anais... Nice, 1993, v. 2, p. 511-518.

MARI, J. J. Psychiatric morbidity in three primary medical care clinics in the city of São Paulo: issues on the mental health of the poor. Social Psychiatry, n. 22, p. 129-138, 1987.

MEHNERT, P.; GRIEFAHN, B.; BRODE, P. A new methodological approach for studies on combined effects of noise and other occupational hazards. In: CONGRESS NOISE AS A PUBLIC HEALTH PROBLEM, 6., 1993, Nice. Anais... Nice, 1993, v. 2, p. 277.

MIKKELSEN, S. A cohort study of disability pension and death among painters with special regard to disabling presenile dementia as an occupational disease. Scand. J. Social Med., supl. 16, p. 34-43, 1980.
MOEN, B. E.; HOLLUND, B. E. Exposure to organic solvents among car painters in Bergen, Norway. Ann. Occup. Hyg., n. 3, p. 185-189, 2000.

MORATA, T. C.; LEMASTERS, G. K. Considerações epidemiológicas para o estudo de perdas auditivas ocupacionais. In: NUDELMANN, A. A. et al. (Orgs.). PAIR. Rio de Janeiro: Revinter, 2001. v. 2, p. 1-16.

NIKOLOV, Z. Hearing reduction caused by manganese and noise. J. F. R. Oto Rhino-Laryngol Audiophonol Chir Maxililoc., n. 23, p. 231-234, 1974.

O'FLLYNN, R. R.; MONKMAN, S. M.; WALDRON, H. A. Organic solvents and presenile dementia: a case referent study using death certificates. Brit. J. Ind. Med., n. 44, p. 259-262, 1987.

OLSEN, J.; SABROE, S.A. A case-referent study of neuropsychiatric disorders among workers exposed to solvents in the Danish wood and furniture industry. Scand. J. Social Med., supl. 16, p. 44-49, 1980.

ORELLANA, H. S.; SALLATO, A. Sistema nervoso (doenças neurológicas e comportamentais ocupacionais). In: MENDES R. (Ed.). Patologia do Trabalho. Rio de Janeiro: Atheneu, 1995. p. 269-285.

ÖSTERBERG, K. et al. A comparison of neuropsychological tests for the assessment of chronic toxic encephalopathy. Am. J. Ind. Med., n. 38, p. 666-680, 2000a.

Psychological test performance

during experimental challenge to toluene and n-butyl acetate in cases of solvent-induced toxic encephalopathy. Scand. J. Work Environ. Health, v. 26, n. 3, p. 219-226, 2000 b.

PENAYO, U.; KULLGREN, G.; CALDERA, T. Mental disorders among primary health care patients in Nicaragua. Acta Psych. Scandin., n. 82, p. 82-85, 1990.

RAMOS, A. et al. Aspectos psiquiátricos da intoxicação ocupacional pelo mercúrio metálico: relato de um caso clínico. Rev. Bras. Psiq., n. 20, p. 200-204, 1998.

RAMOS, A. A. M.; SILVA FILHO, J. F.. Transtornos neuropsiquiátricos e exposição a solventes. Arq. Bras. Psiquiatr. Neurol. Med. Legal, n. 78, p. 8-13, 2001.

Exposição a pesticidas, atividade laborativa e agravos à saúde. Rev. Med. Minas Gerais, v. 14, n. 1, p. 32-36, 2004.

RENTZSCH, M.; PRESCHER, W.; TOLKSDORF, M. New models, methods of evaluation and design solutions for combined load and strain. Arch. Compl. Environ. Studies, v. 4, n. 3, p. 55-63, 1992.

SASS-KORTSAK, A. M.; COREY, P. M.; ROBERTSON, J. M. C. D. An investigation of the association between exposure to styrene and hearing loss. Ann. Epidemiol., n. 5, v. 1, p. 15-24, 1995.

SINGER, R. M. Neurotoxicity guidebook. New York: Van Nostrand Reinhold, 1990. 
TRIEBIG, G.; HALLERMANN, J. Survey of solvent related chronic encephalopathy as an occupational disease in European countries. Occup. Environ. Med., n. 58, p. 575-581, 2001.

VAN DER HOEK, J. A. F.; VERBERK, M. M.;

HAGEMAN, G. Criteria for solvent-induced chronic toxic encephalopathy a systematic review. Int. Arch. Occup. Environ. Health, n. 73, p. 362-368, 2000.

WOOD, R L.; LIOSSI, C. Long-term

neuropsychological impact of brief occupational exposure to organic solvents. Arch. Clin.

Neuropsychol., v. 20, n. 5, p. 655-665, 2005.

YU, I. T. et al. Occupational exposure to mixtures of organic solvents increasesthe risk of neurological symptms among printing workers in Hong Kong. J. Occup. Environ. Med., v. 4, n. 46, p. 323-330, 2004.

ZAVARIZ, C.; GLINA, D. M. R. Avaliação clíniconeuro-psicológica de trabalhadores expostos a mercúrio metálico em indústria de lâmpadas elétricas. Rev. Saúde Públ., v. 5, n. 26, p. 356-365, 1992. 